Portland State University

PDXScholar

\title{
Modeling Intracranial Fluid Flows and Volumes During Traumatic Brain Injury to Better Understand Pressure Dynamics
}

\author{
Wayne W. Wakeland \\ Portland State University, wakeland@pdx.edu \\ James McNames \\ Portland State University \\ Mateo Aboy \\ Portland State University \\ D. Hollemon \\ Oregon Health \& Science University \\ Brahm Goldstein \\ Oregon Health \& Science University \\ Follow this and additional works at: https://pdxscholar.library.pdx.edu/sysc_fac \\ Part of the Biomedical Commons \\ Let us know how access to this document benefits you.
}

\section{Citation Details}

Wakeland, W., McNames, J., Aboy, M., Hollemon, D., \& Goldstein, B. (2003, September). Modeling intracranial fluid flows and volumes during traumatic brain injury to better understand pressure dynamics. In Engineering in Medicine and Biology Society, 2003. Proceedings of the 25th Annual International Conference of the IEEE (Vol. 1, pp. 402-405). IEEE.

This Post-Print is brought to you for free and open access. It has been accepted for inclusion in Systems Science Faculty Publications and Presentations by an authorized administrator of PDXScholar. Please contact us if we can make this document more accessible: pdxscholar@pdx.edu. 


\title{
MODELING INTRACRANIAL FLUID FLOWS AND VOLUMES DURING TRAUMATIC BRAIN INJURY TO BETTER UNDERSTAND PRESSURE DYNAMICS
}

\author{
W. Wakeland ${ }^{1}$, J. McNames ${ }^{2}$, M. Aboy ${ }^{2}$, D. Hollemon ${ }^{3}$, B. Goldstein ${ }^{3}$ \\ ${ }^{1}$ Systems Science Ph.D. Program, Portland State University, Portland, Oregon, USA \\ ${ }^{2}$ Biomedical Signal Processing Laboratory, Electrical and Computer Engineering, Portland State \\ University, Portland, Oregon, USA \\ ${ }^{3}$ Complex Systems Laboratory, Division of Pediatric Critical Care, Oregon Health \& Science University, \\ Portland, Oregon, USA
}

\begin{abstract}
We describe a computer model of intracranial pressure (ICP) dynamics that evaluates clinical treatment options for elevated ICP during traumatic brain injury (TBI). The model uses fluid volumes as primary state variables and explicitly models fluid flows as well as the resistance, compliance, and pressure associated with each intra- and extracranial compartment (arteries and arterioles, capillary bed, veins, venous sinus, ventricles, and brain parenchyma). The model evaluates clinical events and therapies such as intra- and extra-parenchymal hemorrhage, cerebral edema, cerebrospinal fluid drainage, mannitol administration, head elevation, and mild hyperventilation. The model is able to replicate observed clinical behavior in many cases, including elevated ICP associated with severe cerebral edema following subdural, epidural, or intraparynchemal hematoma. The model also mimics cerebrovascular regulatory mechanisms that are activated during TBI.
\end{abstract}

Keywords-intracranial pressure (ICP), traumatic brain injury (TBI), dynamic modeling, therapeutic modeling.

\section{INTRODUCTION}

Elevated ICP associated with TBI is a major clinical concern. Despite the availability of many treatment options for reducing elevated ICP, poor outcomes still result in many cases due to secondary brain injury. It is clear that we only partially understand the complex processes at work during TBI, and many therapies that are commonly used are based upon pathophysiologic evidence that is either lacking or of questionable significance [1]. For example, questions have been raised about the common practice of elevating the patient's head to lower their ICP [2].

To address these and other concerns, researchers have developed multiple computer models for calculating ICP [3-6]. These models are often expressed as an electrical analog. Differential equations are developed for the pressure at different points within the system. The total volume is constrained as indicated by the Monro-Kellie Doctrine which states that total intracranial volume ([brain volume] + [blood volume] + [cerebrospinal fluid volume] + ["other" volume]) is fixed [7].

Excellent mathematical results and insights into the mechanisms that contribute to elevated ICP been reported for these models, and yet they have not had much influence on clinical practice.

We believe that one reason for this is that most physicians are not comfortable with or do not understand electrical analogies. Furthermore, the underlying dynamics clearly depend on fluid flows and volumes rather than the various pressures that are commonly measured during clinical practice and research. In this paper, we describe an ICP dynamic model that uses fluid volumes as state variables rather than pressures. This leads to several useful insights that are discussed in the following sections.

\section{METHODOLOGY}

\section{A. Model Development}

Our model stipulates that total cranial volume remains nearly constant considering the brain parynchema plus several fluid compartments. The fluid compartments include the arterial blood volume, capillary blood volume, venous blood volume, cerebrospinal fluid (CSF) volume, brain parenchymal volume, and "other" volumes (e.g. epidural hematoma). For most clinical scenarios, brain parenchymal volume is assumed to be constant, as is the case with most other ICP dynamic models. However, in some scenarios, especially those associated with certain types of TBI, the assumption of fixed brain volume is not accurate, such as when cerebral edema has occurred.

Similar to previously published models, cerebral autoregulation is modeled as a feedback loop that causes the vasculature to dilate or constrict, taking into account control limits that are non-linear and asymmetric. Our control logic acts only on the flow of blood from the arterial compartment to the capillary bed. The control logic is proportional and has enough gain that it easily maintains the required flow under normal conditions. However, if the venous or arterial volumes are severely reduced, as is often the case with TBI, the associated nonlinear increases in resistance overwhelm the control logic, leading to a loss of cerebral autoregulation. The nonlinear increase in resistance is due in part to Poiseuille's law, which states that resistance to flow in a vessel is inversely proportional to the vessel radius to the fourth power [2]. 
The model uses fluid volumes in each compartment as state variables and explicitly accounts for the fluid flows through each compartment. This approach is more intuitive and makes it is easier to represent the relevant pathophysiology. Blood pressures are computed from the blood volumes and their respective compliances as shown in Equations 1-3.

$\mathrm{P}_{\mathrm{a} \text { ic }}=\mathrm{ICP}+$ ArterialBloodVol/ArterialCompliance

$\mathrm{P}_{\mathrm{c} \text { ic }}=\mathrm{ICP}+$ CapillaryBloodVol/Capillary Compliance

$\mathrm{P}_{\mathrm{v} \text { ic }}=\mathrm{ICP}+$ VenousBloodVol/Venous Compliance

Where $\mathrm{P}_{\mathrm{a} \text { ic, }} \mathrm{P}_{\mathrm{c} \text { ic, and }} \mathrm{P}_{\mathrm{v} \text { ic }}$ represent the pressures in the intracranial arteries, capillaries, and veins, respectively.

ICP is computed using the total intracranial volume and the pressure volume index (PVI) [6]. The PVI is the additional volume needed to cause a 10 -fold increase in pressure, as shown in Equation 4.

$\mathrm{ICP}=\operatorname{BaseICP} *(10)^{(\text {TotalCranialVolume-BaseCranialvolume }) / P V I}$

Equation 4 indicates that the model is not consistent with the Monro-Kellie Doctrine because small increases in the total intracranial volume are allowed to occur, thereby causing ICP to increase exponentially.

The model was developed using the STELLA [8] simulation language because: 1 ) it is well suited to the formulation phase of dynamic modeling; 2) it is easy to represent the flows and storage of fluids; and, 3) model structure can be easily reviewed and understood by nonmathematicians.

The model has been designed to reproduce the dynamic behavior associated with multiple types of TBI pathophysiology including epidural hematoma, subdural hematoma, intraparynchemal hemorrhage, focal or generalized cerebral edema, and depressed skull fracture. Many previously published ICP models allowed for only a limited number of pathophysiologic scenarios. Our model also allows for various combination of pathophysiologies commonly encountered in clinical practice. For example, the model's response to a simulated epidural hematoma may include focal cerebral edema and elevated ICP, as is frequently observed in clinical situations.

Our model also takes into account different time constants for development of hemorrhage depending on the source of the bleeding (venous vs. arterial vs. capillary). It also incorporates common treatment modalities such as intravenous mannitol, elevating the head of the bed to $30^{\circ}$, mild hyperventilation (decreased $\mathrm{PaCO}_{2}$ ), and cerebrospinal fluid drainage via indwelling intraventricular catheter.

The model diagram is shown in Figure 1 . We recognize that many readers may not be familiar with this type of diagram. The rectangles represent volumes (blood, CSF, brain parynchema), and the double arrows represent flows that change the volumes. In the middle of each double arrow is a symbol representing a valve. The small cloud-like symbols represent model boundaries. When first viewing the diagram, the circles and thin arrows in the diagram may be ignored. Blood flows from the cloud symbol in the upper left quadrant of the model into the rectangle representing the arterial compartment. Blood then flows from the arteries into the capillary compartment, and then from the capillaries into the venous compartment. Blood exits at the cloud symbol in the upper right quadrant of the model.

A tiny amount of blood is synthesized into plasma and then CSF via an ultra-filtration process [2]. CSF circulates, and is then reabsorbed. This is modeled as CSF flowing out of the cranial vault. CSF may also be drained via an indwelling intraventricular catheter placed for that purpose and for measuring ICP in some patients with severe TBI. The final rectangle is the brain volume, which might increase due to swelling.

Now consider the thin arrows and circles. The thin arrows that connect into a particular valve or circle indicate the information needed to compute the flow rate or the value of the variable. Circles represent additional equations or logic. For example, "Pa ic" (pressure, arterial, intracranial) is represented as a circle, indicating that it is an algebraic formula. Three arrows point into $\mathrm{Pa}$ ic, indicating that it is computed [instantaneously] from three other model components: Arterial Compliance, Arterial Blood Volume, and ICP.

The model diagram is detailed and complex, but the advantage of this complexity is that the logic is made very explicit.

\section{B. Model Behavior}

Behavior is simulated by numerically integrating the underlying differential equations. Accurate integration is required due to the high flow rates in comparison to the volumes in the reservoirs. STELLA can do this, but is nevertheless very limited in this regard, providing as its most powerful integration algorithm the $4^{\text {th }}$ Order RungeKutta with fixed step size.

\section{Clinical Reference Data}

CSF drainage is a common therapy for reducing elevated ICP in severe TBI. Real-time ICP signal data from three episodes of CSF drainage were obtained from the Complex Systems Laboratory [9], including 5 minutes prior to the drainage and 15 minutes afterwards. Since the signal integrity is compromised when the drain is first opened, a small segment of data is intentionally omitted at the point when the drainage was initiated. The data was sampled at $125 \mathrm{~Hz}$. The signal was lowpass filtered and decimated by 100 to an effective sample rate of $1.25 \mathrm{~Hz}$. This eliminated the pulsatile component of the signal, but retained the trend. Figure 2 shows this data in the time domain for three specific episodes. 


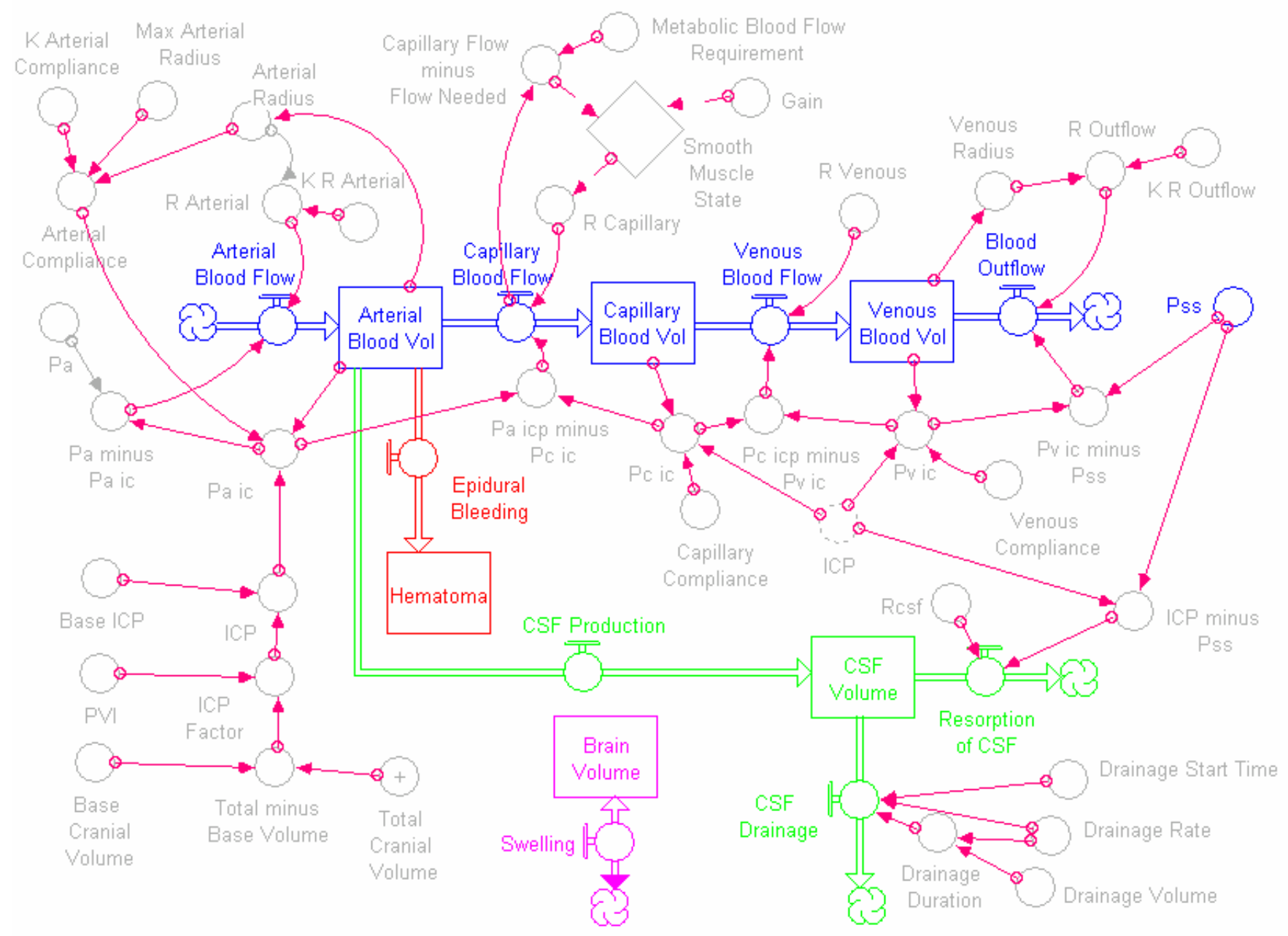

Figure 1: Structure of the ICP dynamic model. Abbreviations are as follows: CSF, cerebrospinal fluid; ICP, intracranial pressure; K, constant; Max, maximum; Pa, arterial blood pressure; Pa ic, intracranial arterial pressure; Pc ic, intracranial capillary pressure; Pv ic, intracranial ventricular pressure; Pss, saggital sinus pressure; PVI, pressure-volume index; R, resistance; R Arterial, arterial resistance; Rcsf, resistance to CSF re-absorption. Other variable names are spelled out in order to more clearly indicate what they represent. Note that cerebral edema can be simulated by increasing the contents of the reservoir named Brain Volume via the flow named Swelling; an epidural hematoma can be simulated by allowing blood to flow from the reservoir named Arterial Blood Vol to the reservoir named Hematoma; and similarly for other combinations of pathologies.

\section{EXAMPLES}

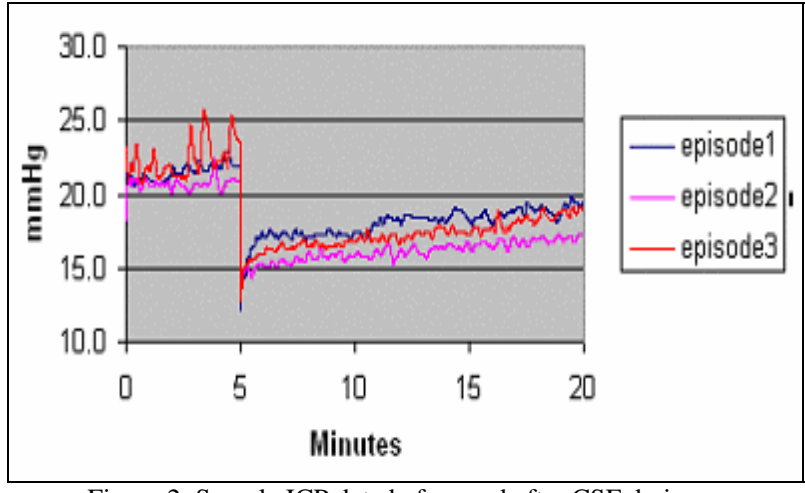

Figure 2: Sample ICP data before and after CSF drainage

\section{A. Example Model Run}

Figure 3 shows an example simulation run four minutes in duration. During the first minute, the model indicates dynamic equilibrium. Then, from 1 to 1.5 minutes, a $25 \mathrm{~mL}$ epidural hematoma is simulated. This causes ICP to increase, and both arterial and venous blood are forced out of the cranial vault. From 2 to 2.3 minutes, CSF fluid drainage is simulated in order to reduce ICP. The overall ICP time dynamics computed by the model are correct in a qualitative sense.

\section{B. Application to Calibration of Model to Clinical Data}

The model was calibrated to approximate the clinical data in Figure 2. In order to replicate this behavior, the model 


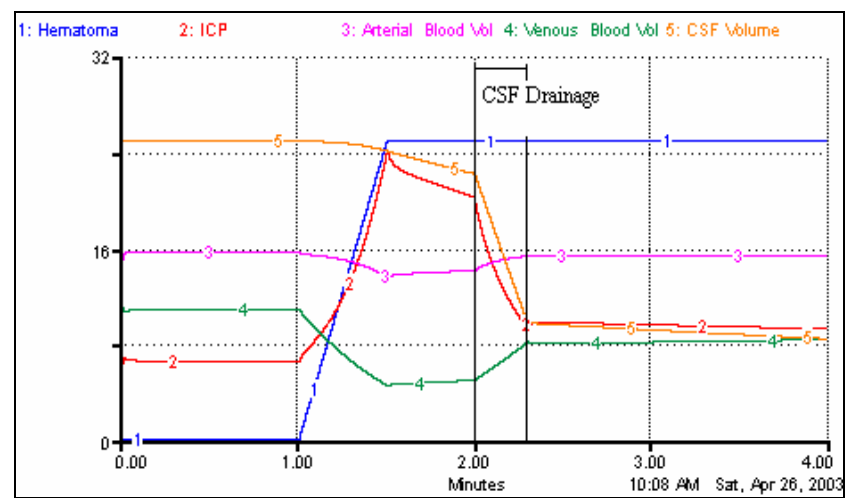

Figure 3: Base simulation run of the ICP dynamic model. The vertical axis show volumes in units of $\mathrm{mL}$ and ICP in units of $\mathrm{mmHG}$.

estimated a $24 \mathrm{~mL}$ epidural hematoma, CSF drainage of $6.5 \mathrm{~mL}$, and an increase in the resistance to CSF uptake (such as that seen with blockage of CSF circulation or resorption). Figure 4 shows the ICP predicted by model versus the average values for the three clinical episodes.

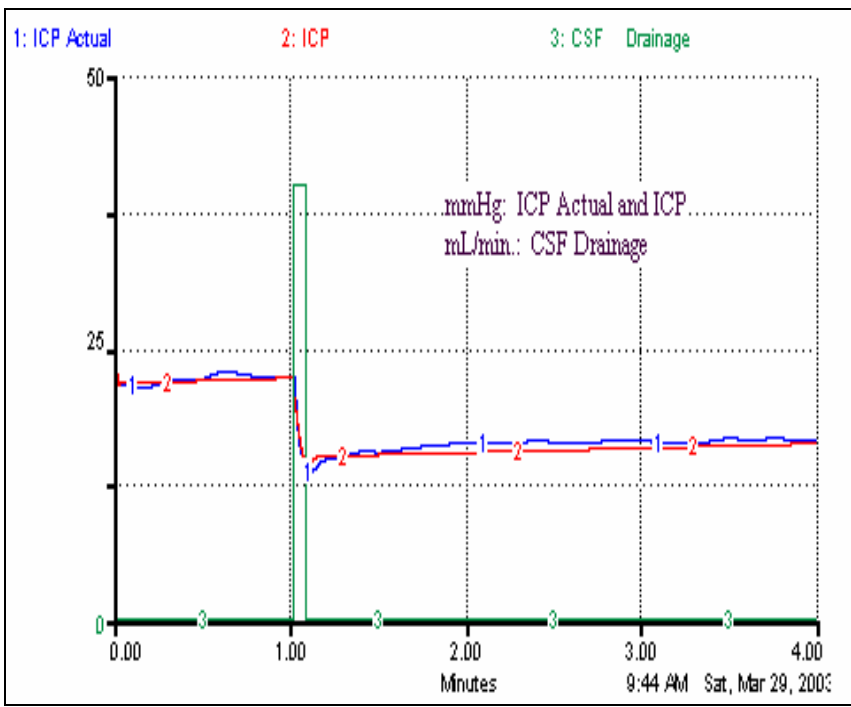

Figure 4: ICP before and after CSF drainage at 1 minute, Model (red line) vs. Actual Data (blue line) (y axis units $=\mathrm{mmHg}$ )

In Figure 4, time 0.00 is one minute prior to the drain. In both the actual data and the model, the drainage begins at time 1.00. In the model, the drainage occurs over a 5 second period. The actual ICP data during the drainage is invalid, and therefore is discarded. For plotting purposes, the actual data during the drainage period is synthesized using a negative exponential fit between the two endpoints

In this particular clinical case, the exact pathophysiologic changes that caused elevated ICP are not known, but the model enabled the researchers to explore multiple potential etiologies. The ICP values calculated by the model closely match the actual ICP data.

\section{CONCLUSION}

The behavior of our initial model appears to be qualitatively correct in cases such as those discussed above. We are now beginning to calibrate the model quantitatively against data that have been carefully collected and clinically annotated in order to synchronize events such as clinical interventions with the signal data being recorded.

Possible refinements to our approach include the use of mathematical optimization to select parameter values, enhancing the cerebral autoregulation logic, and modeling CSF circulation. As the model is refined, it will be rigorously tested as prescribed in the system dynamics literature [10].

\section{ACKNOWLEGEMENT}

This research was supported in part by grants from the Northwest Health Foundation and the Friends of Doernbecher.

\section{REFERENCES}

[1] P. Adelson, S. Bratton, N. Carney, R. Chesnut, H. DuCourdray, B. Goldstein, et al. Guidelines for the acute management of severe traumatic brain injury in infants, children and adolescents. Crit Care Med 2003 and $J$ Trauma 2003 (in press).

[2] M. Rosner. Pathophysiology and management of increased intracranial pressure. In: Neurosurgical Intensive Care, B.T. Andrew (ed), McGraw-Hill, Inc, 1993: 57-112.

[3] M. Czonyka, S. Piechnik, H. Richards, P. Kirkpatrick, P Smielewski, J. Pickard. Contribution of mathematical modeling to the interpretation of bedside tests of cerebrovascular autoregulation. J Neurosurg 1997;63:721731.

[4] M. Ursino, M. Iezzi, N Stocchetti. Intracanial Pressure Dynamics in Patients with Acute Brain Damage: A Critical Analysis with the Aid of a Mathematical Model. IEEE Transactions on Biomedical Engineering 1995;42:529-540.

[5] M. Ursino, C. Lodi, S. Rossi, N Stocchetti. Intracranial pressure dynamics in patients with acute brain damage. J Appl Physiol 1997;82:12701282.

[6] A. Marmarou, K. Shulman, R. Rosende. A nonlinear analysis of compliance and outflow resistance of the cerebrospinal fluid system. $J$ Neurosurg 1978;48:332-344.

[7] J. Miller. Normal and increased intracranial pressure. In: J. Miller (ed) Northfield's Surgery of the Central Nervous System. $2^{\text {nd }}$ ed. Edinburg, Blackwell. 1987. ch. 2.

[8] http://www.hps-inc.com/STELLAVPSR.htm. 3/23/03.

[9] http://www.ohsuhealth.com/dch/complex. 3/23/03.

[10] J. Sterman. Business Dynamics: Systems Thinking and Modeling for a Complex World. Irwin/McGraw Hill, Boston, MA 2000. 